\title{
A Model for Operator Endpoint Stiffness Prediction During Physical Human-Robot Interaction
}

\author{
Antonio Moualeu and Jun Ueda \\ Bio-Robotics \& Human Modeling Laboratory, Georgia Institute of \\ Technology, Atlanta, GA
}

Physical contact established during interaction between a human operator and a haptic device creates a coupled system with stability and performance characteristics different than its individual subsystems taken in isolation. Proper incorporation of operator dynamics in physical human-robot interaction ( $\mathrm{pHRI}$ ) conditions requires knowledge of system variables and parameters, some of which are not directly measurable. Operator endpoint impedance, for instance, cannot be directly measured in typical haptic control conditions. Several endpoint impedance estimation techniques have been explored in previous literature, based on measured kinematics and/or other correlated metrics. Arm muscle activity, measured through surface electromyography (sEMG), has been used in previous literature to estimate endpoint stiffness, which is the static component of impedance. Co-activation (co-contraction) of antagonistic arm muscles forming a pair around a joint is known to be the driving factor in modulation of endpoint stiffness. However, previous work employing muscle co-contraction to predict endpoint stiffness has mainly been absent, due in part to the inefficacy of operator models to incorporate muscle co-activation into the prediction scheme.

The current study proposes a method for prediction of operator endpoint stiffness based on measured co-contraction levels of a select group of muscles. The proposed methodology incorporates an upper extremity musculoskeletal model that accounts for muscle redundancy and the role of muscle co-contraction on arm stiffness modulation. The study hypothesizes that a free parameter, currently known in the literature to represent the nullspace of the mapping between muscle forces and joint torques, is a random variable of which probability density function can be estimated. Changes in this parameter directly affect changes in operator endpoint stiffness.

Ten healthy subjects were asked to resist perturbations induced by a one degree of freedom (DOF) haptic paddle device while measurements, including muscle activities of four arm muscles, were being carried out. Direct derivation of stiffness values at the endpoint was compared to simulated endpoint stiffness values obtained using the proposed predictive methodology.

Ten out of forty prediction trials resulted in a statistically significant correlation between predicted and actual stiffness values. Impressive stiffness prediction results, with over $99 \%$ peak accuracy in value, were found in only one trial using a combination of the proposed method along with a standard static optimization method for muscle force computation. Though the possibility of using a probabilistic approach to stiffness prediction was shown, robustness and generalizability of the proposed approach to multi-DOF systems remain to be addressed.

Keywords: physical human-robot interaction, endpoint stiffness, co-contraction, prediction, model

Authors retain copyright and grant the Journal of Human-Robot Interaction right of first publication with the work simultaneously licensed under a Creative Commons Attribution License that allows others to share the work with an acknowledgement of the work's authorship and initial publication in this journal.

Journal of Human-Robot Interaction, Vol. 4, No. 3, 2015, Pages 170-193, DOI 10.5898/JHRI.4.3.Moualeu 


\section{Introduction}

Recent shifts in robotic research and development have been influenced by a push toward the realization of "co-robots," true robotic partners that work beside, or cooperatively with, people. The next generation of robots is expected to operate smoothly and effectively in flexible and changing environments, acting in direct support of and in a collaborative relationship with human operators. These flexible environments include automotive and aircraft fabrication processes, which often produce subassemblies that must ultimately be fitted, in spite of minor dimensional errors. Such relaxed tolerance requirements constitute the main motivation behind the current interest in novel shared control approaches aimed toward increasing human capability while allowing the operator to focus their mental capacity on adapting to changes in the environment.

Developing shared control strategies aimed at improving performance in pHRI partly requires an in-depth understanding of static and dynamic properties of the new system established as a result of the physical coupling between human and robotic device. These properties differ from those of each isolated system prior to the interaction. Hence, accurate modeling of the coupling between robot and operator dynamics, as well as its bilateral nature, is critical to reaching greater stability and performance during interaction. Current control approaches have come short of achieving these goals, mainly due to inefficient adjustment of controller gains to changes in interaction port impedance, which is a critical measure of pHRI systems performance.

Typical haptic controllers usually incorporate an impedance controller in order to mask actual system dynamics through an assistive force, in combination with a position controller to minimize error between current and desired (subscript $d$ ) endpoint positions. Fig. 1 summarizes the sdomain block diagram of a haptic control system combining robot (subscript $h$ ) and operator (subscript $o$ ) dynamics. Gallagher (2013) provides full derivation of the system equations found in the figure and a simple root-locus analysis of stability showing that the system is susceptible to greater instability as a result of increases in endpoint stiffness and/or control loop time delay. A robust control strategy that accounts for stochastic variations of the stiffness parameter $k_{O}$ is worth exploring and is one of the overarching goals of this research.

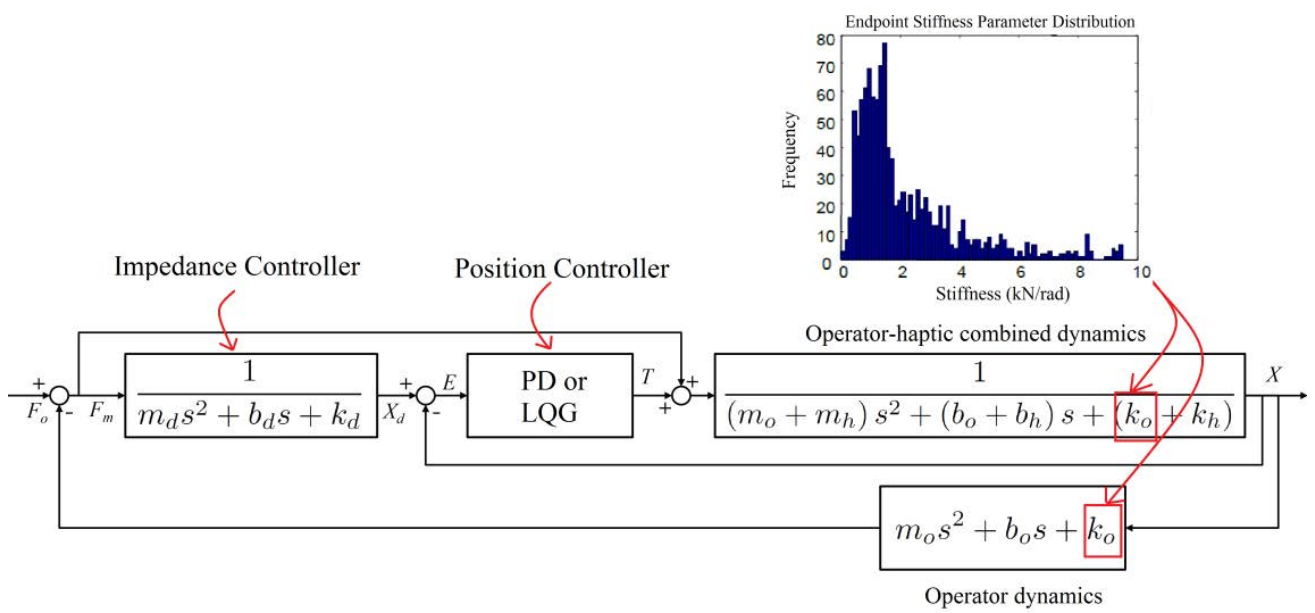

Figure 1. Continuous-domain block diagram of a haptic control system. Typical haptic systems combine an impedance controller with an inner position controller. Accurate characterization of plant dynamics at the interaction port requires the combination of operator and haptic dynamics. The operator stiffness $k_{o}$ is a stochastically varying parameter that we hypothesize to be predictable. 
One of the main challenges faced in designing novel control schemes for a pHRI system is the difficulty in directly measuring impedance at the interaction point. This challenge has reduced current control methods to estimation techniques based on correlated metrics, with significant amounts of error and variance reported in previous literature. Previous studies, for example, have used muscle activity, measured through electromyography (EMG), for stiffness estimation. Current understanding of neuromechanics suggests that the human central nervous system (CNS), consisting of the brain and spinal cord, is able to change endpoint stiffness levels by modulating co-activation (co-contraction) levels across agonist and antagonistic muscles around joints (Gribble, Mullin, Cothros, \& Mattar, 2003). Muscles spanning the same side of a joint are referred to as agonist muscles, whereas muscles on opposite sides of a joint are antagonistic muscles. Modulation of endpoint stiffness is mainly driven by simultaneous activation (i.e., co-contraction) of antagonistic muscles. However, given the greater number of muscle actuators compared to the number of joints in the human body (i.e., muscle redundancy), a unique set of muscle forces cannot be directly derived from knowledge of endpoint force (Bernstein, 1967).

In this paper, we present a novel approach combining probabilistic prediction with an endpoint stiffness estimation method (Hu, Murray, \& Perreault, 2011) based on musculoskeletal modeling. The new method utilizes an integrated model (Ueda, Ming, Krishnamoorthy, Shinohara, \& Ogasawara, 2010) that can characterize the coupling between the human musculoskeletal system and robot dynamics at the level of individual muscles. Endpoint stiffness results obtained from simulations are compared to offline experimental measurements of endpoint stiffness obtained during quasi-static perturbation trials using a 1-DOF haptic device. The estimation approach presented in this paper is part of a larger proposed research focused on two aims: (1) understanding pHRI in a bilateral rather than unilateral manner and (2) predicting human physical state using a probabilistic approach based on physiological measures. Knowledge acquired from aims (1) and (2) can be integrated into a shared human-robot interaction control structure that meets the needs of a human operator. Though this paper only focuses on aim (2), success in the overall proposed research would benefit communities interested in the adaptive shared control approach for advanced manufacturing and process design, including automobile, aerospace, and construction industries. For instance, a variety of car assembly or other industrial tasks, such as pick-and-place tasks, peg-in-hole insertion, painting, palletizing, or installing engines/doors, can be evaluated in our proposed research using a robotic device conceptually shown (Fig. 2). Task performance could be evaluated using metrics such as speed or accuracy in task completion and interaction port behavior (subjective interaction "feel" reported by the operator).

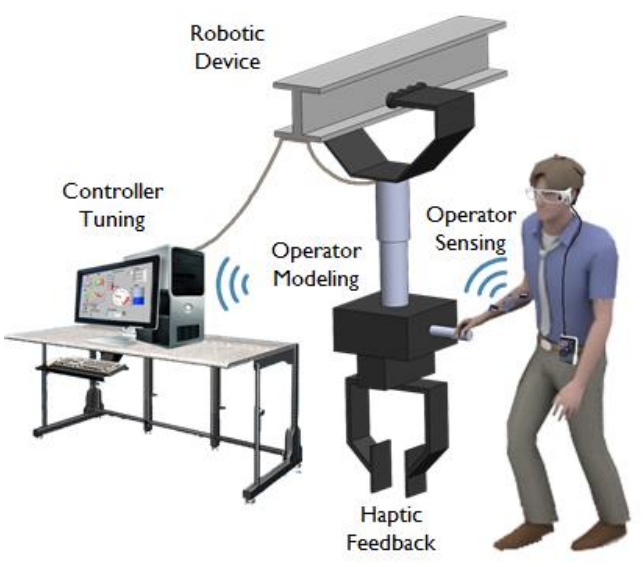

Figure 2. Industrial tasks can be evaluated in our overall proposed research study, though not covered in the current paper, using a conceptually shown robotic device with controller tuning based on estimated operator modeling. 


\section{Background and Problem Statement}

\section{Prior Research on Mechanical Impedance Estimation}

Human stiffness measurement through movement perturbations applied with a robotic device has a long history (Mussa-Ivaldi, Hogan, \& Bizzi, 1985; Tsuji \& Kaneko, 1996; Burdet et al., 2000; Perreault, Kirsch, \& Crago, 2001, 2002). Mussa-Ivaldi et al. (1985) developed the first reported experimental method capable of characterizing human hand stiffness both numerically (as a matrix) and graphically (as an ellipse). They also showed that static endpoint stiffness properties are highly dependent on arm configuration. The effect of arm posture on arm impedance characteristics has subsequently been studied more recently for a single endpoint position (Krutky, Ravichandran, Trumbower, \& Perreault, 2010; Krutky, Trumbower, \& Perreault, 2013).

Since Hogan's 1984 study, theories about how the human CNS is able to regulate mechanical impedance characteristics at the endpoint have emerged over the last few decades in the field of neuromechanics. This emergence has occurred mostly through investigation of the effect of muscle activity on limb stiffness, the static component of impedance, during planar tasks (Flash \& MussaIvaldi, 1990; Tsuji \& Kaneko, 1996; Gomi \& Osu, 1998; Franklin \& Milner, 2003). They have led to the current state of understanding stipulating that the CNS regulates co-contraction, and hence limb stiffness, in order to facilitate movement accuracy. Results from studies conducted by Gribble et al. (2003), for instance, are consistent with this current hypothesis. Also, findings from Darainy, Malfait, Gribble, Towhidkhah, and Ostry (2004) were shown to be consistent with the idea that arm stiffness is controlled through the use of at least two independent co-contraction commands. More recently, Patel, O'Neill, and Artemiadis (2014) investigated the three-dimensional (3D) case and found an anisotropic increase of arm stiffness due to muscle co-contraction, underlying the directional tuning of endpoint stiffness to introduced instability, as demonstrated in previous literature for lower dimensions (Franklin et al., 2007).

\section{Role of Muscle Activation in Endpoint Stiffness Estimation}

Short-Range Muscle Stiffness. While previous studies have investigated changes in endpoint stiffness characteristics in relation to muscle activity and specifically muscle co-contraction, very few have actually directly assessed the muscle properties responsible for stiffness modulation of an entire limb. Osu et al. (2002), for instance, related joint torque and stiffness in both static and dynamic tasks to a novel method of evaluating muscle co-contraction levels around the joint, using EMG signals. Using this novel approach, they were able to determine that the viscoelasticity in the musculoskeletal model contributes more when internal models are inaccurate, whereas internal models play a major role after completion of task learning. However, the method for endpoint stiffness is not based on any EMG measurement, requires multiple trials, and is used to infer joint stiffness.

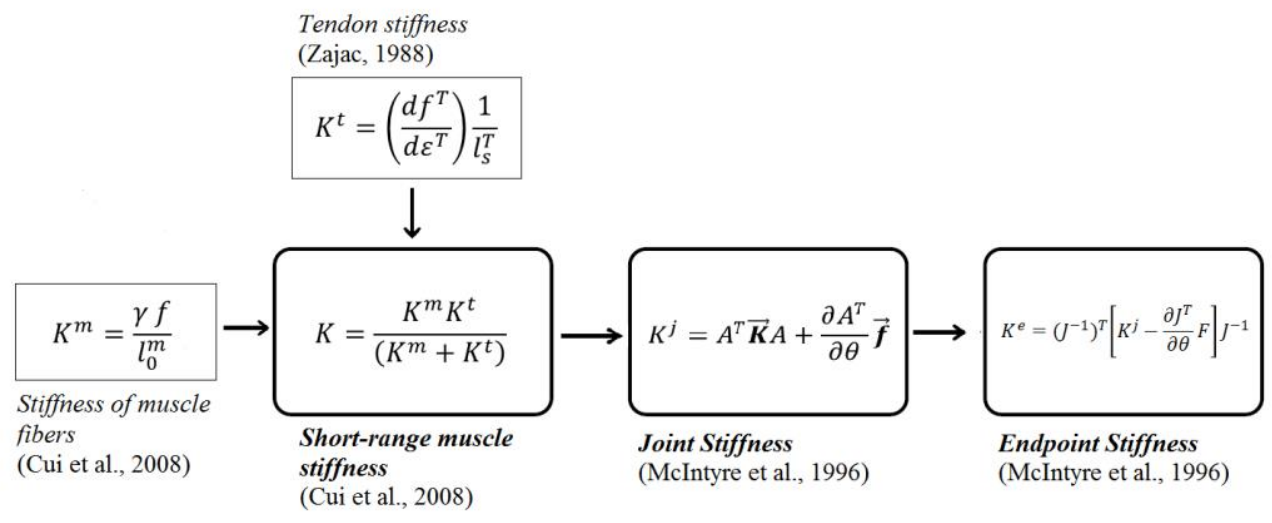

Figure 3. Summary of endpoint stiffness estimation from muscle activity (Hu et al., 2011). Muscle short-range stiffness is suggested to be a major contributor to endpoint stiffness of the human arm, and it can be estimated based on muscle geometry and active force generated. 
Results from a study by $\mathrm{Hu}$ et al. (2011) suggest that muscle-short range stiffness can be used to estimate endpoint stiffness of the human arm, instead of the commonly used muscle forcelength curve, yielding greater accuracy in stiffness orientation, shape, and area. The study used a musculoskeletal model of the upper limb consisting of 37 muscle segments and incorporating kinematic representations of the shoulder and elbow joints. They concluded that the intrinsic stiffness from individual muscles is a major contributor to endpoint stiffness. Fig. 3 provides a summary of the estimation method. Below is the related nomenclature.

$\begin{array}{ll}A: & \text { muscle moment arm matrix of muscles } \\ \frac{d f^{T}}{d \varepsilon^{T}}: & \text { slope of the dimensionless tendon force-strain curve } \\ f: & \text { muscle force } \\ \overrightarrow{\boldsymbol{f}}: & \text { column vector of all muscle forces in the model } \\ F: & \text { endpoint force vector } \\ \gamma: & \text { dimensionless scaling constant }(=23.4 \text { in Hu et al. (2011) paper) } \\ J: & \text { Jacobian matrix whose transpose }\left(J^{T}\right) \text { maps endpoint force to joint torques } \\ K: & \text { short-range muscle stiffness } \\ \overrightarrow{\boldsymbol{K}}: & \text { diagonal matrix with short-range muscle stiffness values along diagonal and } \\ K^{e}: & \text { endpoint stiffness matrix } \\ K^{j}: & \text { joint stiffness matrix } \\ K^{m}: & \text { muscle fiber stiffness } \\ K^{t}: & \text { tendon stiffness } \\ l_{0}^{m}: & \text { optimal fiber length at maximum activation } \\ l_{s}^{T}: & \text { tendon slack length } \\ \theta: & \text { joint angle vector (i.e., posture) }\end{array}$

Most of the variables and parameters above are either computed or obtained from previous literature. Among these variables, the origins of the muscle force vector $\overrightarrow{\boldsymbol{f}}$ and of the dimensionless scaling constant $\gamma$ are of most interest for this paper.

Muscle force estimation. Static optimization methods are used to determine individual muscle forces in biomechanical modeling due to the redundant nature of the musculoskeletal system. The most common method comes from Crowninshield and Brand (1981); nominal human muscle force $\boldsymbol{f}_{\mathbf{0}}$ is determined through minimization of a physiologically based optimality criterion, as shown in Eq. (1).

$$
\begin{aligned}
& u(\boldsymbol{f})=\sum_{j=1}^{N}\left(\frac{f_{j}}{\operatorname{PCSA}_{j}}\right)^{r} \rightarrow \min \\
& \text { subject to }\left\{\begin{array}{c}
\boldsymbol{\tau}_{\boldsymbol{h}}=A \boldsymbol{f} \\
f_{\min _{j}} \leq f_{j} \leq f_{\max _{j}}(j=1, \ldots, N)
\end{array}\right.
\end{aligned}
$$

where $u(f)$ - optimality criterion

$f_{j}$ - muscle force of the $j$-th muscle, with $f_{\min _{j}}$ and $f_{\max _{j}}$ its minimum and maximum

$\boldsymbol{\tau}_{\boldsymbol{h}}-$ human joint torque vector

$\mathrm{PCSA}_{j}$ - physiological cross sectional area (PCSA) of the $j$-th muscle

$r-$ integer power $($ possible values $=[2,3,4, \ldots])$

$\tau_{h}-$ human joint torque vector

$N$ - number of muscles in the model 
This cost function, however, fails to predict muscle co-contraction of antagonistic muscles and has proven to be inappropriate for tasks where limb stiffness is modulated without corresponding changes in joint torque (Collins, 1995; Gomi \& Osu, 1998; Milner, Cloutier, Leger, \& Franklin, 1995). Hu et al. (2011) reported low values in elbow stiffness predicted by their model during an extension task, for example, and attributed this discrepancy to the failure in cocontraction prediction. The invisibility of muscle co-contraction in the joint space can be characterized by Eq. (2).

$$
\boldsymbol{f}=\boldsymbol{f}_{\mathbf{0}}+\left(\boldsymbol{I}-\boldsymbol{A}^{+} \boldsymbol{A}\right) \boldsymbol{\beta}(t)
$$

where $\boldsymbol{f}$-actual muscle force vector

$\boldsymbol{f}_{\mathbf{0}}-$ nominal muscle force vector

$\boldsymbol{I}$ - identity matrix

$\boldsymbol{A}^{+}$- pseudo-inverse of moment arm matrix $A$

$\boldsymbol{\beta}(t)$ - variable representing kernel of mapping between muscle forces and joint torques

\section{Problem Statement: Probabilistic Prediction of Muscle Coordination}

In theory, knowing the values of the $\boldsymbol{\beta}(t)$ vector at each time sample would allow a musculoskeletal model to accurately estimate individual muscle forces contributing to endpoint forces. A change in $\boldsymbol{\beta}(t)$ changes the human operator's arm stiffness, hence affecting the overall stability and performance of a pHRI system. The current study hypothesizes that the parameter $\boldsymbol{\beta}(t)$ is a random variable whose mean and variance are dependent on operator intent. Its probability density function (PDF) can be predicted by means of Markov models. We expect that muscle activities measured by EMG correlate with $\boldsymbol{\beta}(t)$. Probabilistic switching models should then predict muscle coordination, including co-contraction, from the observation of movement sequences.

Yet, given the multivariate nature of the variable, direct prediction of $\boldsymbol{\beta}(t)$ is by no means an easy endeavor. Using the model employed by $\mathrm{Hu}$ et al. (2011), for instance, would require having to deal with 37 features. Therefore, it is necessary to find a workaround the computational burden of prediction through dimensionality reduction techniques, or simply through observation of a much lower dimension variable capable of directly affecting the value of $\boldsymbol{\beta}(t)$.

Cui, Perreault, Maas, and Sandercock (2008) estimated muscle short-range stiffness using a muscle model of a cat hindlimb. The model equations, shown in Fig. 3 above, include computation of muscle force $f$ as a function of muscle fiber stiffness $K^{m}$, optimal muscle fiber length at maximum activation $l_{0}^{m}$, and a dimensionless scaling constant $\gamma$ with constant value of 23.4 (in $\mathrm{Hu}$ et al., 2011). Given a similar assumption under static conditions, the change in muscle force vector from nominal levels can be used to extract a relationship between $\boldsymbol{\beta}(t)$ and $\gamma$, as shown in Eq. (3).

$$
\Delta \boldsymbol{f}=\boldsymbol{f}-\boldsymbol{f}_{\mathbf{0}}=\frac{1}{\gamma} \Delta\left(\boldsymbol{K}^{\boldsymbol{m}} \boldsymbol{l}_{\mathbf{0}}^{m}\right) \overrightarrow{\mathbf{1}}=\left(\boldsymbol{I}-\boldsymbol{A}^{+} \boldsymbol{A}\right) \boldsymbol{\beta}(t)
$$

where $\boldsymbol{K}^{\boldsymbol{m}}$ - diagonal matrix with muscle stiffness $K^{m}$ along diagonal and zero in other entries

$l_{\mathbf{0}}^{m}$ - diagonal matrix with muscle fiber length $l_{0}^{m}$ along diagonal and zero in other entries

$\overrightarrow{\mathbf{1}}$ - column vector with all entries of 1

$\Delta-$ difference between true (current) value and nominal value of a variable

Unlike $\mathrm{Hu}$ et al. (2011), this paper proposes that $\gamma$ is also a time-dependent variable, related to the $\boldsymbol{\beta}(t)$ variable at each time point and configuration by Eq. (4). As described by the equation, changes in $\gamma(t)$ at a given configuration affect changes in $\boldsymbol{\beta}(t)$, and therefore, endpoint impedance. Similarly, its PDF could also be estimated through Markov models.

$$
\boldsymbol{\beta}(t)=\frac{1}{\gamma(t)}\left[\left(\boldsymbol{I}-\boldsymbol{A}^{+} \boldsymbol{A}\right)^{-1} \Delta\left(\boldsymbol{K}^{\boldsymbol{m}} \boldsymbol{l}_{\mathbf{0}}^{m}\right) \overrightarrow{\mathbf{1}}\right]
$$

Hidden Markov Models. There is a long history of using a Bayesian approach to estimate the intent of an operator interacting with a device (Yamada, Umetani, Daitoh, \& Sakai, 1999; Schmid, 
Weede, \& Worn, 2007; Takeda, Hirata, \& Kosuge, 2007). As a probabilistic approach, it allows for reasoning under uncertainty. Since the variables of interest (e.g., $\boldsymbol{\beta}(t), \gamma(t))$ in our study are not directly measurable, hidden Markov models (HMMs) seem to be the natural choice for a predictive model. These models have shown to be easy to train and to use for inference; they can handle continuous data and usually impose less computational burden (Oliver \& Horvitz, 2005). Other predictive models, such as dynamic Bayesian networks (DBNs), Artificial Neural Networks (ANNs), and support vector machines (SVMs) could also be explored but may incur more training data and computation time.

An HMM is a doubly Markov (stochastic) process with an underlying stochastic process that is hidden and can only be observed through another set of stochastic processes (Rabiner \& Juang, 1986). A Markov process, as illustrated in Eq. (5), describes the probability of being in a current state (at time $t$ ) as a function of the preceding state only (Rabiner, 1989).

$$
\begin{aligned}
P\left[q_{t}\right. & \left.=S_{j} \mid q_{t-1}=S_{i}, q_{t-2}=S_{k}, \ldots\right] \\
& =P\left[q_{t}=S_{j} \mid q_{t-1}=S_{i}\right]
\end{aligned}
$$

where $q_{t}$ - actual state at time $t$

$S$ - discrete state of the Markov chain

An HMM uses a transition model $T$ and a sensor model $O$ to perform estimation of a current, hidden-state $X_{t}$ from a list of possible discrete states $S_{1} \ldots S_{N}$, based on the preceding state $X_{t-1}$ and a currently observed metric $Y_{t} . T$ is an $N \times N$ matrix of probabilities where each row represents probabilities for a single previous state and each column a current state. $O$ is an $N \times D$ matrix, where $D$ represents the number of possible discrete observations. The model handles continuous data by grouping them into discrete bins with known PDFs; the index to each bin is then used to train the model. Fig. 4 displays a representation of an HMM for the case of the current study.

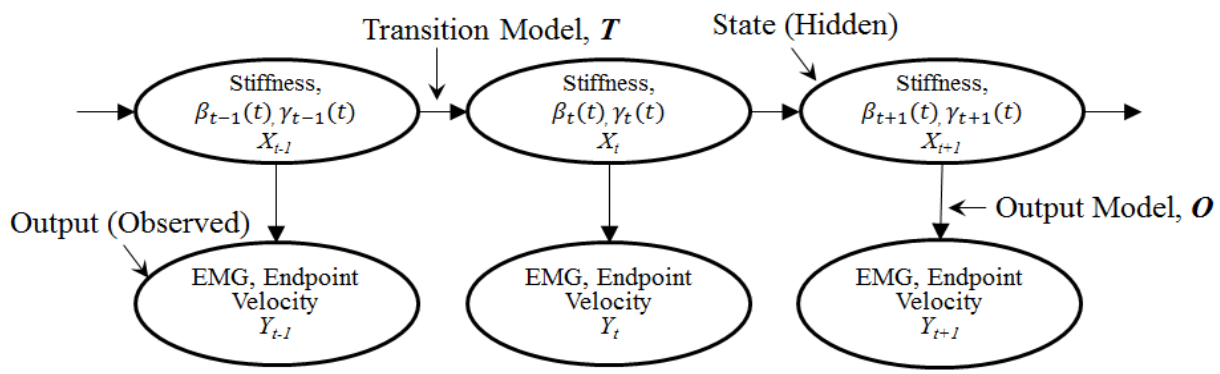

Figure 4. Representation of stiffness prediction model using HMM. In this study, four EMGs producing two co-contraction signals, in addition to an endpoint velocity signal, are all observed variables. Endpoint stiffness, the main hidden variable, can be derived through musculoskeletal modeling, using either $\beta(t)$ or $\gamma(t)$ as hidden-state variables.

\section{Materials and Methods}

\section{Quasi-Static Stiffness Measurement Through Perturbation Experiment}

All tests conducted in this study were performed using a 1-DOF haptic paddle device $(0.305 \mathrm{~m}$ radius to handle), shown in Fig. 5, which can produce a maximum force of $100 \mathrm{~N}$ at the handle, has a bandwidth of $10 \mathrm{~Hz}$, and a range of motion of $\pm 0.325 \mathrm{rad}( \pm 18$ degrees). The system setup includes a multi-axis force/torque sensor (ATI Industrial Automation, Apex, NC) with stand-alone controller and a brushless DC motor (Anaheim Automation, Inc., Anaheim, CA) linked to a quadrature optical encoder (US Digital, Vancouver, WA), controlled by a servo motor drive (Pacific Scientific, CA). The haptic device is run using a CompactRio real-time controller, 
programmable and deployable through a user interface in LabView software (National Instruments, Austin, TX). EMG measurements were acquired using a wireless EMG system (Cometa Srl, Milan, Italy) with true differential electrodes (common-mode rejection ratio > 120 $\mathrm{dB}$ ) without a reference electrode. Raw EMG signal is amplified by a gain of 1000, sampled at a 2 $\mathrm{KHz}$ rate by 16-bit resolution analog-to-digital converters and band-pass filtered between 10 and $500 \mathrm{~Hz}$. A user interface program was developed in LabView to conduct these experiments.

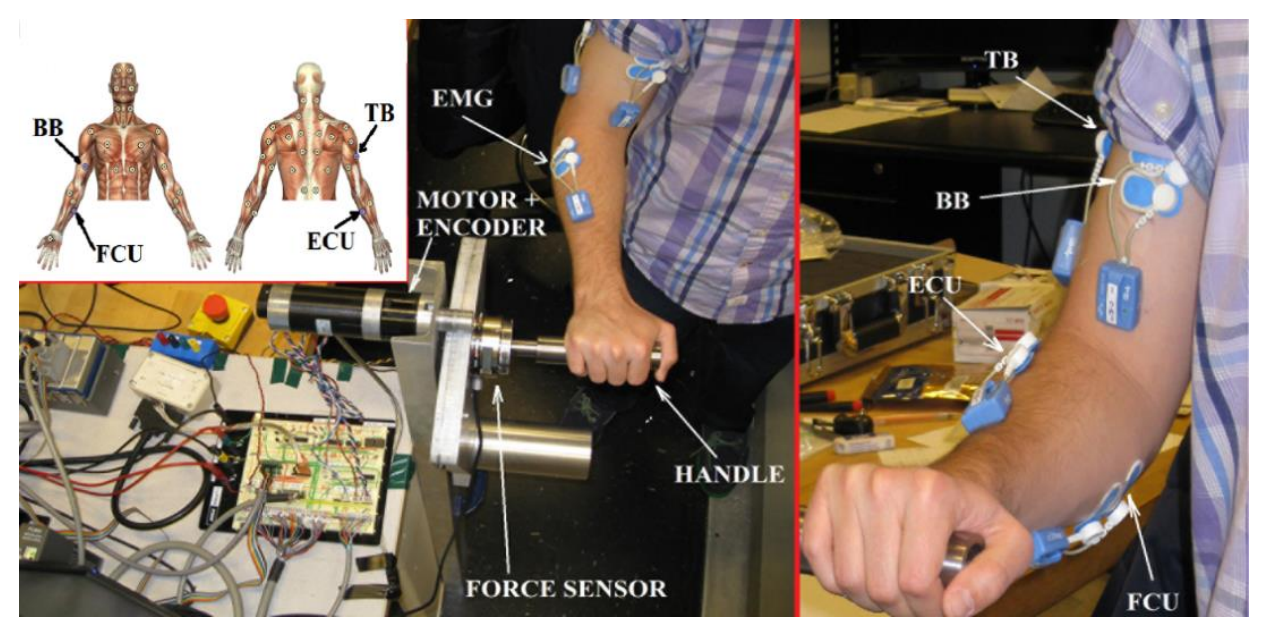

Figure 5. Experimental setup and EMG placement. Ten subjects were asked to resist force perturbation applied at the handle using uniformly distributed and randomly generated amplitude $(0-40 \mathrm{~N})$, hold/duration (0.5-1.5 s), and timing between perturbations $(2-20 \mathrm{~s})$.

A total of 10 participants (7 male, 3 female; age range 21-31; all subjects were right-handed) were told to maintain the device steady while a command force, of amplitude between 0 and $40 \mathrm{~N}$, was generated at the contact interface using a random generator with uniform distribution. The direction of the command force (forward or backward) was also randomly changed according to a uniform distribution. The timing between perturbations was varied between 2 and $20 \mathrm{~s}$, whereas the duration (hold time) of each force perturbation was varied between 0.5 and $1.5 \mathrm{~s}$, using a random number generator with uniform distribution as well. The participants were required to immediately apply a restoring force upon perturbation and relax once the desired return position was reached. The initial (and return) position corresponds to arm configuration II in Fig. 6.

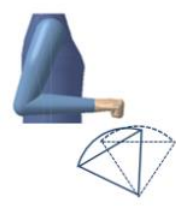

I. $20^{\circ}$ shoulder extension

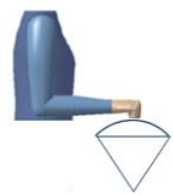

II. $0^{\circ}$

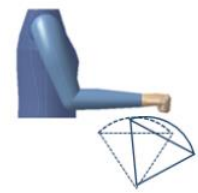

III. $20^{\circ}$ shoulder flexion

Figure 6. Three examples of arm configuration. During perturbation experiments in this study, configuration II corresponded to the initial (and return) configuration. Assuming that the forearm remains horizontal during all motions, shoulder and elbow joint angles can be geometrically derived from endpoint position, as seen in Eq. (9), avoiding the need for an arm configuration tracking system. 
Subjects were asked to pick and maintain a relaxed standing position as well as keep their wrist straight throughout the experiment. Stiffness values were directly calculated from the force and displacement (from the initial position) signals collected as shown in Eq. (6). Each subject underwent a single trial experiment that lasted several minutes, with an average of 15 perturbations (repetitions). Hence, a single plot of quasi-static stiffness time history was generated per subject and used for both training data and validation (testing) data extraction.

$$
K(t)=\frac{F(t)}{\Delta x}=\frac{F(t)}{\left(\phi(\mathrm{t})-\phi_{\mathrm{o}}\right) R}
$$

where $F(t)$ - measured endpoint force at time $t$

$\phi(t)$ - measured endpoint (encoder) position at time $t$

$R$ - haptic paddle radius from center to handle $(=0.305 \mathrm{~m})$

Muscle activity was acquired through EMG signals from two arm and two forearm antagonistic muscle pairs. These muscles are the biceps brachii (BB), the triceps brachii (TB), which form the first antagonistic pair, and the flexor carpi Ulnaris (FCU) and extensor carpi Ulnaris (ECU), all highlighted in Fig. 5. These muscles were chosen among a group of candidate muscles spanning the elbow and the wrist joints in a validation study (Gallagher, 2013), based on their level of antagonism, assessed using the angle between their moment arm vectors. Two perfectly antagonistic muscles, which act in an opposite fashion about the same joint, should have an angle of antagonism of 180 degrees between their respective moment arm vectors. Muscle cocontractions of the first and second muscle pairs are abbreviated in this paper as $\mathrm{BB} / \mathrm{TB}$ and FCU/ECU, respectively.

EMG signal processing included DC component removal, full-wave rectification, and lowpass filtering $(1.5 \mathrm{~Hz})$ to obtain the linear envelope. For each antagonistic pair of muscles $(i, j)$, processed EMG values $\left(f_{i}(t), f_{j}(t)\right)$ are scaled to respective maximum recorded EMG values $\left(f_{i}^{\text {max }}\right.$, $\left.f_{j}^{\max }\right)$ to obtain normalized muscle activity $\left(f_{i}^{\%}(t), f_{j}^{\%}(t)\right)$ values between 0 and 1 . Eq. (7) displays the co-contraction index, $c c_{i, j}$, a measure of muscle co-contraction determined by taking the minimum normalized muscle activity for a muscle pair at each sample time (Gallagher et al., 2012; Gribble et al., 2003; Thoroughman \& Shadmehr, 1999).

$$
c c_{i, j}(t)=\min \left(f_{i}^{\%}(t), f_{j}^{\%}(t)\right)
$$

All measurements acquired (endpoint force, endpoint kinematics, and four EMG measurements) and directly computed variables during these experimental trials were subsequently used as simulation inputs, in order to compare and evaluate different prediction tools.

\section{Stiffness Prediction Model}

Musculoskeletal Modeling. An integrated model that can characterize the coupling between the human musculoskeletal system and robot dynamics at the level of individual muscles has been developed by Ueda et al. (2010). This upper-right musculoskeletal model is a 5-rigid link model with 12 joints from the waist to the wrist and 51 arm muscles. Notably different from this model, maximum isometric force for each muscle is posture dependent in the Hu et al. (2011) model. The 
Hu et al. (2011) model also accounts for pennation angle, and the optimal fiber length computation is dependent on activation, which is time-dependent and computed in a different manner. For a human model with $M$ joints and $N$ muscles, the relationship between joint torques and endpoint force in a typical pHRI system is given by Eq. (8), under static assumptions and neglected dynamics of both robot and human bodies.

$$
\boldsymbol{\tau}_{\boldsymbol{h}}=J^{T}(\boldsymbol{\theta}) \boldsymbol{F}(=A \boldsymbol{f})
$$

In order to investigate one of the study's main claim, the Ueda et al. (2010) model described above was used in a previous preliminary study (Moualeu, Gallagher, \& Ueda, 2014) to generate, purely through simulation in Matlab software, a dataset of random values of the $\boldsymbol{\beta}(t)$ vector variable, subject to muscle force feasibility constraints (see Eq. (1)), using a random number generator with uniform distribution. A total of 5000 vectors were obtained from computation at 5 varying endpoint positions $\left(-20^{\circ},-10^{\circ}, 0^{\circ}, 10^{\circ}\right.$ and $\left.20^{\circ}\right)$ and 20 endpoint force levels between 5 and $100 \mathrm{~N}$. Hence, for each combination of endpoint position and endpoint force, 50 data vectors (repetitions) were generated. The purpose of this previously carried out simulation was to generate a data pool that could be used for possible grid search and training purposes. No experimental data were acquired in the preliminary study.

Arm configuration in this study, specifically shoulder rotation, elbow flexion/extension, and wrist pronation/supination, were calculated using encoder values (if experimental data were used as input) or endpoint position (for pure simulations), as shown in Eq. (9).

$$
\begin{gathered}
s=\phi(\mathrm{rad}) \\
e=\frac{\pi}{2}+\phi(\mathrm{rad}) \\
w=\frac{4 \pi}{9} \mathrm{rad}
\end{gathered}
$$

where $s$-shoulder rotation angle

$e$ - elbow angle

$w$ - wrist pronation angle

All other entries in the joint angle vector $\boldsymbol{\theta}$ were zero. Muscle forces, and therefore cocontraction values, were computed. Endpoint stiffness values could subsequently be computed using the procedure outlined in Fig. 3. Values for several parameters, including optimal fiber length and tendon slack length parameters (Holzbaur, Murray, \& Delp, 2005; Garner \& Pandy, 2003), slope of the tendon force-strain curve $(=34.375$, heuristic value agreeing with Maganaris \& Paul, 2002; Magnusson, Aagaard, Rosager, Dyhre-Poulsen, \& Kjaer, 2001), and dimensionless scaling constant $\gamma$ of 23.4, as suggested in Hu et al. (2011), were all obtained from literature.

As previously stated, the current study focuses on improving the origination process of the two following variables in the previously outlined $\mathrm{Hu}$ et al. (2011) method for endpoint stiffness prediction: the muscle force vector $\boldsymbol{f}$ and the dimensionless scaling variable $\gamma(t)$.

Comparison of Two Muscle Force Computation Methods. For the muscle force vector computation, two methods were evaluated, using measurements acquired during the perturbation experiments: (1) the first method uses the standard optimality principle from Crowninshield and Brand (1981); (2) the second and proposed method performs a grid search through the pool of 
$\boldsymbol{\beta}(\boldsymbol{t})$ values previously generated (Moualeu et al., 2014), accounting for feasibility as well as boundary constraints imposed by muscle activity (BB/TB and FCU/ECU co-contraction indexes) measurements experimentally obtained. The narrowed $\boldsymbol{\beta}(\boldsymbol{t})$ pool is used to pick the highest probable vector value, based on its norm distribution. The vector value picked is used to compute the muscle force vector using Eq. (2). These two methods are referred to as the nominal method and the beta method, respectively.

Probabilistic prediction of $\gamma(\boldsymbol{t})$ using $\mathbf{H M M s}$. Two proposed calibration methods were evaluated in order to test our main claim about the variability of $\gamma(t)$. (1) The first method performs a calibration procedure yielding a single optimal scaling value, using a grid search method during which endpoint stiffness values directly obtained from the experiments are compared to estimated values using the Hu et al. (2011) method. This constant value, obtained by averaging optimal values computed at each time point during the simulation, is later used for prediction purposes. (2) The second method uses three HMM predictors, each with a single observed process: two co-contraction sequences $\mathrm{BB} / \mathrm{TB}$ and FCU/ECU and one endpoint velocity sequence (see Fig. 4). The state sequence for all three models corresponds to $\gamma(t)$ values obtained at each time point using the same grid search process from the first method. Additionally, a decision HMM is used to determine which observation (BB/TB, FCU/ECU or velocity) should be used to infer the current state, based on the previous state result. Since we are dealing with continuous variables, all sequences were discretized using bins with similar PDFs. A uniform standard deviation was calculated by dividing the standard deviation of each sequence by the length of the sequence. For all trained HMMs, the initial state probabilities were obtained using a random number generator with uniform distribution; parameters, such as tolerance and maximum number of iterations, were all kept constant across all data training. The HMMs were used to estimate the optimal $\gamma(t)$ at each time point. The two methods are referred to as the constant method and the HMM method, respectively.

The four methods for computation of muscle forces (nominal and beta method) and the dimensionless scaling variable $\gamma(\mathrm{t})$ (constant and HMM method) were combined during simulations. As previously mentioned, the role of the calibration process is to generate either a sequence or a single optimal scaling constant that is later used during prediction model testing. An experimental procedure summary is provided in the flow diagram shown in Fig. 7. Fig. 8 displays a typical time history of a single subject's one-dimensional endpoint stiffness directly obtained experimentally from perturbation trial data, using Eq. (6). Data collection during each one-time subject experiment usually lasted 5-10 minutes, but only a small time window (ms-s range) was used for simulation purposes (training and validation). The data collection sampling frequency varied between experiments, due to the fact that data transmission by means of user datagram protocol/transmission control protocol (UDP/TCP) used in this study was not guaranteed. The average sampling time was found to be $76.3 \mathrm{~Hz}( \pm 11.8 \mathrm{~Hz})$. The time windows were carefully chosen so as to include transient changes in stiffness, in order to assess the efficacy of the prediction method being evaluated. In order to extract a time window, whether for training or prediction, a stiffness peak was identified and 100 data points were taken around the peak (24 samples before peak and 75 after peak). Based on the average sampling frequency, a window corresponded to about $1.31 \mathrm{~s}(1.13 \mathrm{~s}-1.55 \mathrm{~s})$ of data. Also, as displayed in Fig. 8, the dataset used for training corresponded to the time window with the highest recorded stiffness value. By using the largest stiffness range possible for training, the probability of a predicted stiffness value falling outside of the training stiffness range is minimal. Each time window chosen for HMM model training was resampled to 500 time data points. Resampling subsequent to training was done for the following reasons: 1) HMM training required a large enough training data size, and 2) the computation time for calibration procedure (grid search) performed to obtained the HMM training set of $\gamma(t)$ was already high (hours long) with 100 data points. 


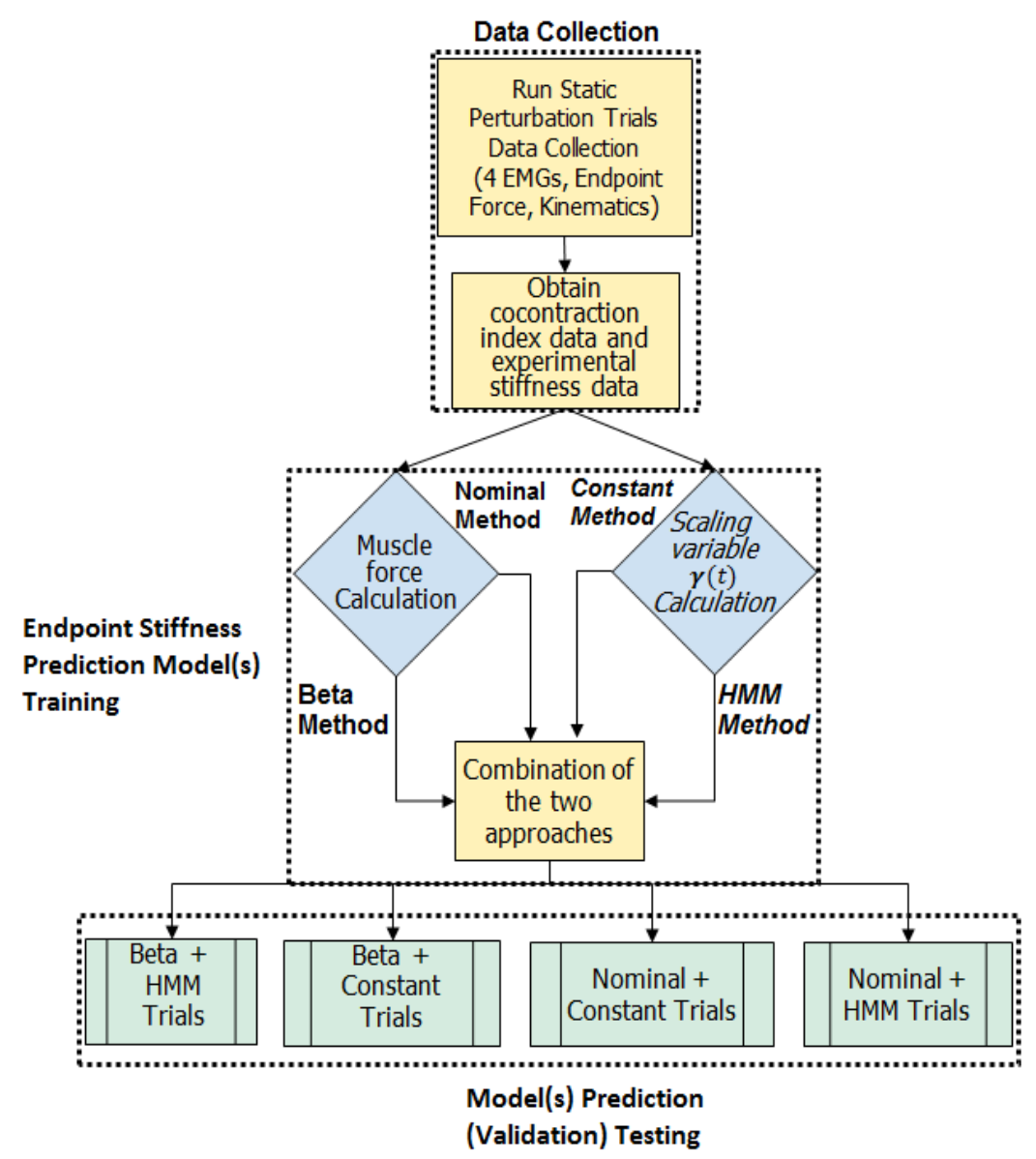

Figure 7. Flow diagram of the experimental procedure for this study. During static perturbation trials, EMG, endpoint force, and kinematical data are collected, in order to generate muscle co-contraction, endpoint velocity, and experimental stiffness data. Training of stiffness prediction models stems from combinations of two approaches: muscle force determination and muscle stiffness scaling variable (or constant) $\gamma(t)$ determination in a musculoskeletal model of the arm. A different dataset or time window (see Fig. 8) is then used in validation simulation using the trained prediction model. The predicted stiffness result is compared to the selected experimental dataset using correlation analysis. Hence, for each subject, four training models were obtained and four corresponding stiffness predictions were carried out.

\section{Results and Discussion}

Comparison of $\beta$ Generated Muscle Force Distributions to Experimental Distributions in a Subject

Distributions of feasible muscle force vector norms, generated at different configurations and endpoint forces using randomly generated $\boldsymbol{\beta}(\boldsymbol{t})$ values, were compared to single subjects' 
experimentally obtained distributions, based on EMG measurements, in the four muscles (BB, TB, FCU, and ECU) used throughout this study.

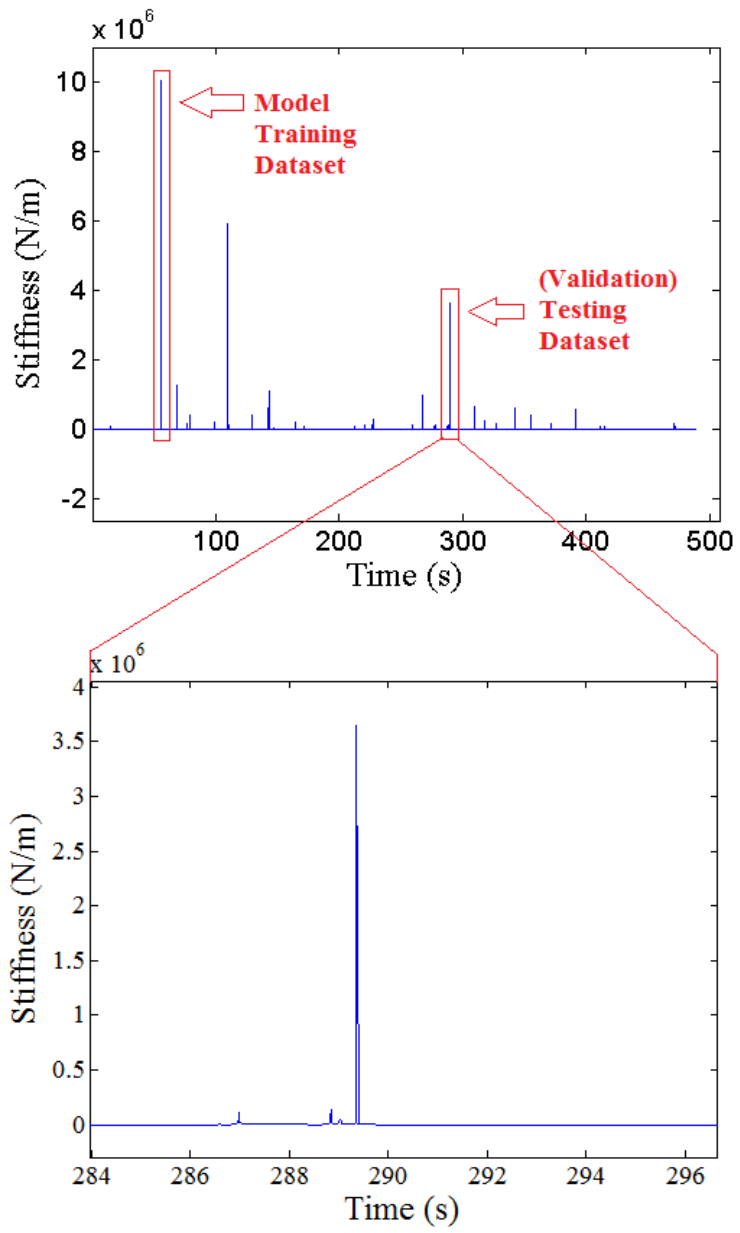

Figure 8. (Top) Time history of one-dimensional (1D) quasi-static stiffness, estimated experimentally for a single subject. The plot is obtained from endpoint force and encoder position data using Eq. (6). The training dataset always had a higher recorded stiffness peak value than the dataset used for validation. (Bottom) Close-up plot of stiffness response from a single perturbation.

The results, presented in Fig. 9, revealed that simulated forces for some of the muscles (FCU, BB, and TB) were significantly lower $(\mathrm{p}<0.05)$ than the experimental force levels obtained for the subject. These results seem to underlie a common issue of underestimation encountered in most musculoskeletal models, which can partly be attributed to the cost function associated with the optimization approach used to determine the muscle forces. These low muscle force estimates can ultimately affect endpoint stiffness estimation levels. Hu et al. (2011), for instance, reported low values in elbow stiffness predicted by their model during extension tasks. Inaccurate estimation of endpoint stiffness values in the specific case pHRI can mean inaccurate adjustment of control gains, which can ultimately lead to instability, poor performance, or in some cases, dangerous 
1.

(a)
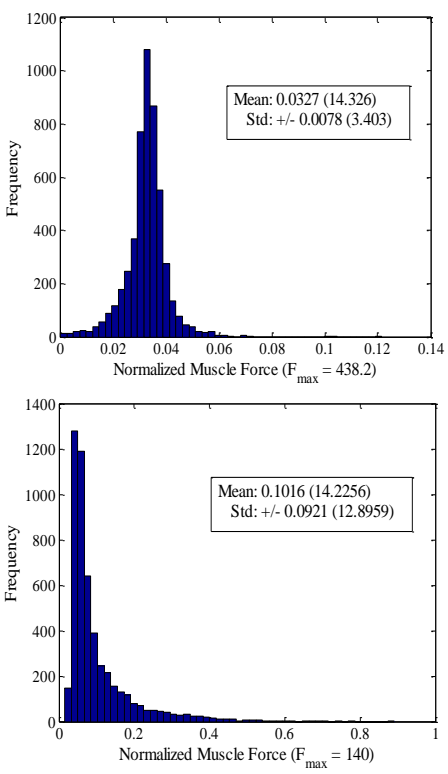

(b)

(c)
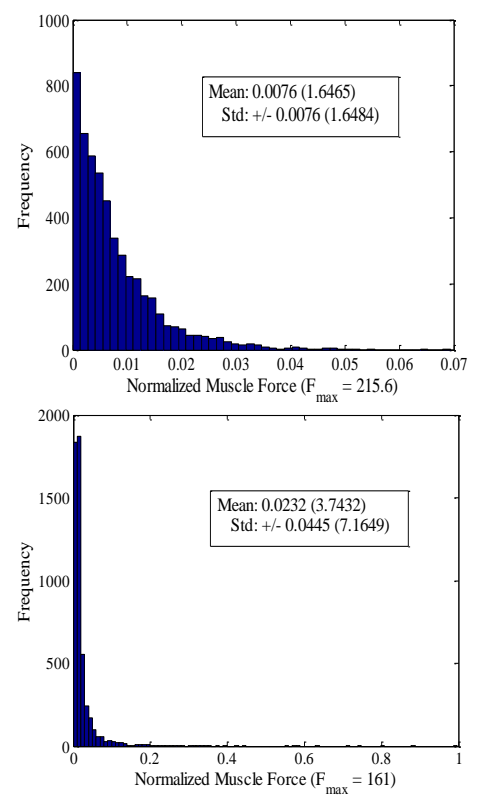

2.
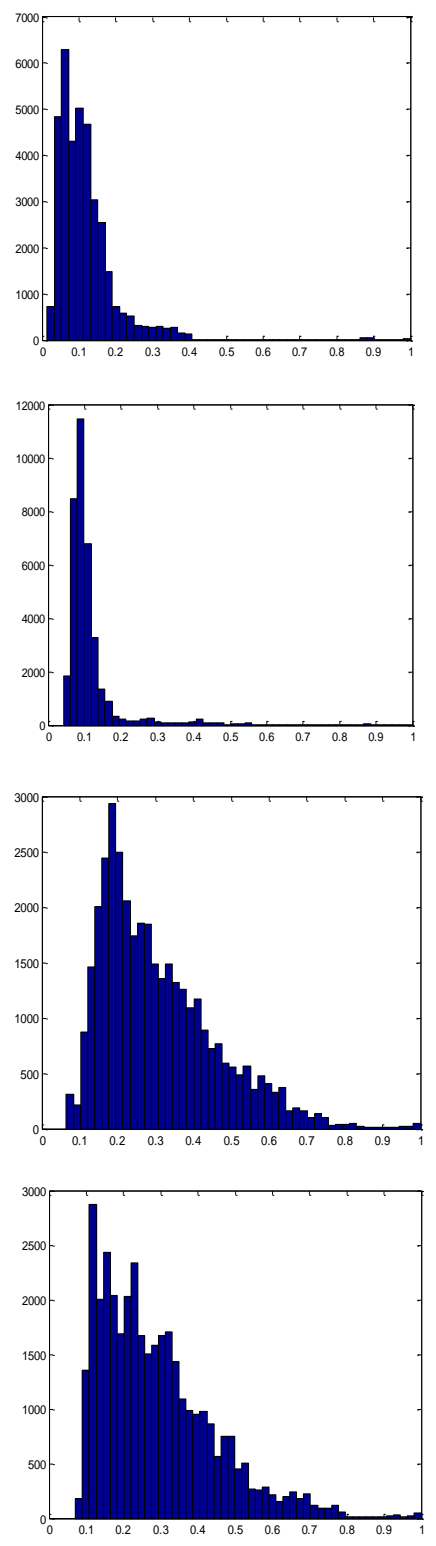

Figure 9. Comparison of histogram distributions between simulated (left) normalized muscle forces obtained using $\beta(\mathrm{t})$ estimation and experimental (right) values from a single subject. 9(a) FCU muscle, $a_{1}$ - simulated, $a_{2}$ - experimental. 9(b) ECU muscle, $b_{1}$-simulated, $b_{2}$-experimental. 9(c) BB muscle, $c_{1}$ simulated, $c_{2}$ - experimental. 9(d) FCU muscle, $d_{1}$ - simulated, $d_{2}$ - experimental. For all graphs, muscle force (in Newtons) is normalized with respect to a maximal value ( $\mathbf{F}_{\max }$ in Newtons), and thus shown here as a unitless value between 0 and 1.

conditions if the robotic device is tuned based on absolute impedance values. Given both intersubject and intra-subject variability in muscle activity patterns and endpoint stiffness levels depending on task, intent, and several other parameters, scalability of musculoskeletal models is crucial to achieve proper calibration prior to use for inference purposes. 


\section{Probabilistic Prediction of Transient Changes in Muscle Stiffness}

Fig. 10 presents a comparison between peak stiffness values obtained through prediction and the actual peak stiffness values for each type of trial and each subject in this study. Overall, the

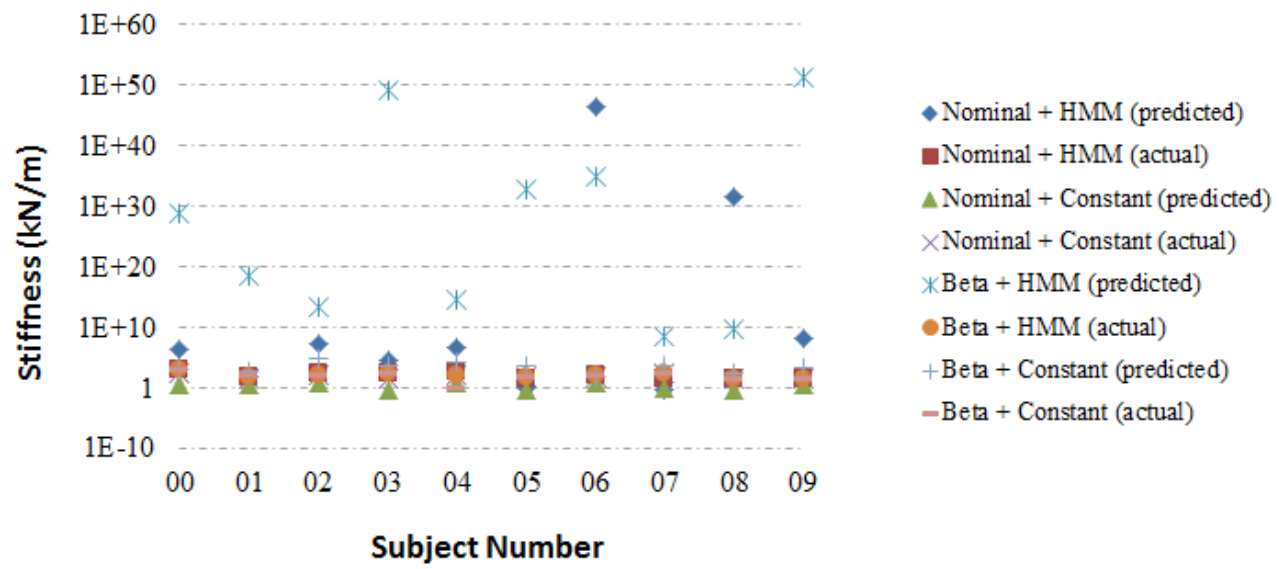

Figure 10. Comparison between predicted and actual stiffness peak values for each type of trial and each subject in the current study.

greatest discrepancies between prediction and experimental values were found in trials involving the HMM method, with the largest differences coming from the beta $+H M M$ methods. Results of the endpoint estimation can be categorized into either failed attempts or successful attempts, based solely on estimation of absolute stiffness values and correlation analysis of the data. Table 1 provides a summary of correlation coefficients between experimental and predicted stiffness, following each of the four combined calibration methods during data analysis for each subject.

Table 1. Summary of the correlation between experimentally determined stiffness and predicted stiffness for all combinations of muscle force determination and scaling variable determination approaches. Statistically significant correlation values are highlighted in bold, with $\mathrm{p}$ values given, corresponding to successful trials.

\begin{tabular}{|c|c|c|c|c|c|c|c|c|c|c|}
\hline $\begin{array}{l}\text { Subject } \\
\text { Number }\end{array}$ & 00 & 01 & 02 & 03 & 04 & 05 & 06 & 07 & 08 & 09 \\
\hline $\begin{array}{l}\text { Nominal } \\
+H M M\end{array}$ & -0.0076 & $\begin{array}{l}0.2561 \\
(p<0.05)\end{array}$ & -0.020 & $\begin{array}{l}0.4683 \\
(p<0.01)\end{array}$ & 0.1635 & -0.0832 & -0.0310 & 0.0821 & -0.0223 & -0.0056 \\
\hline $\begin{array}{c}\text { Nominal } \\
+ \\
\text { Constant }\end{array}$ & -0.0036 & $\begin{array}{c}\mathbf{0 . 3 3 1 1} \\
(\mathbf{p}<0.01)\end{array}$ & $\begin{array}{l}-0.2998 \\
(\mathrm{p}<0.01)\end{array}$ & $\begin{array}{l}\mathbf{0 . 2 9 4 6} \\
(\mathrm{p}<0.01)\end{array}$ & $\begin{array}{l}-0.2143 \\
(p<0.05)\end{array}$ & -0.0654 & $\begin{array}{l}-0.2230 \\
(\mathrm{p}<0.05)\end{array}$ & -0.0603 & $\begin{array}{l}-\mathbf{- 0 . 3 0 5 2} \\
(\mathbf{p}<0.01)\end{array}$ & $\begin{array}{l}0.5464 \\
(p<0.01)\end{array}$ \\
\hline $\begin{array}{c}\text { Beta }+ \\
H M M\end{array}$ & -0.0109 & -0.0237 & -0.0169 & -0.0185 & -0.0090 & -0.0421 & -0.0299 & $\begin{array}{c}\mathbf{0 . 8 2 8 1} \\
(\mathrm{p}<0.01)\end{array}$ & -0.0251 & -0.0129 \\
\hline $\begin{array}{c}\text { Beta }+ \\
\text { Constant }\end{array}$ & -0.0176 & -0.0593 & -0.0166 & -0.0194 & -0.0177 & -0.0482 & 0.0146 & -0.0526 & -0.0369 & -0.0021 \\
\hline
\end{tabular}


Table 2 provides a summary of averaged correlation values for each combined calibration method, with the ranges provided. At first glance of Table 1, we can immediately observe that for scaling variable $\gamma$ determination, the constant method, used by Hu et al. (2011), yields the highest number of statistically significant correlations between predicted endpoint stiffness and actual endpoint stiffness. For muscle force determination, the nominal method, also used by $\mathrm{Hu}$ et al., yielded better correlation overall, compared to the beta method. Some negative correlations were found, though $\mathrm{p}$ values for most of those correlations suggest there is essentially zero correlation between

Table 2. Summary of population average correlations between predicted endpoint stiffness and experimentally determined stiffness values.

\begin{tabular}{|c|c|c|c|c|}
\hline & Nominal + HMM & Nominal + Constant & Beta + HMM & Beta+Constant \\
\hline $\begin{array}{c}\text { Average } \\
\text { Correlation } \\
\text { [Range }]\end{array}$ & $\begin{array}{c}0.0800 \\
{[-0.0832,0.4683]}\end{array}$ & $\begin{array}{c}0.0001 \\
{[-0.3052,0.5464]}\end{array}$ & $\begin{array}{c}0.0639 \\
{[-0.0421,0.8281]}\end{array}$ & $\begin{array}{c}-0.0256 \\
{[-0.0593,0.0146]}\end{array}$ \\
\hline $\begin{array}{c}\text { Average } p \\
\text { value } \\
\text { Range }]\end{array}$ & $\begin{array}{c}0.5266 \\
{[8.979 \mathrm{E}-07,0.9555]}\end{array}$ & $\begin{array}{c}0.2107 \\
{[4.0791 \mathrm{E}-04,0.9714]}\end{array}$ & $\begin{array}{c}0.7531 \\
{[2.2708 \mathrm{E}-26,0.9296]}\end{array}$ & $\begin{array}{c}0.7820 \\
{[0.5579,0.9833]}\end{array}$ \\
\hline
\end{tabular}

predicted and actual stiffness values. Overall, 10 out of 40 total trials yielded statistically significant results, whereas most prediction attempts were considered to be failed attempts, one of which is depicted in Fig. 11. However, results obtained from one specific subject (subject 01) have provided some eye-opening insights and are explained in more detail below.

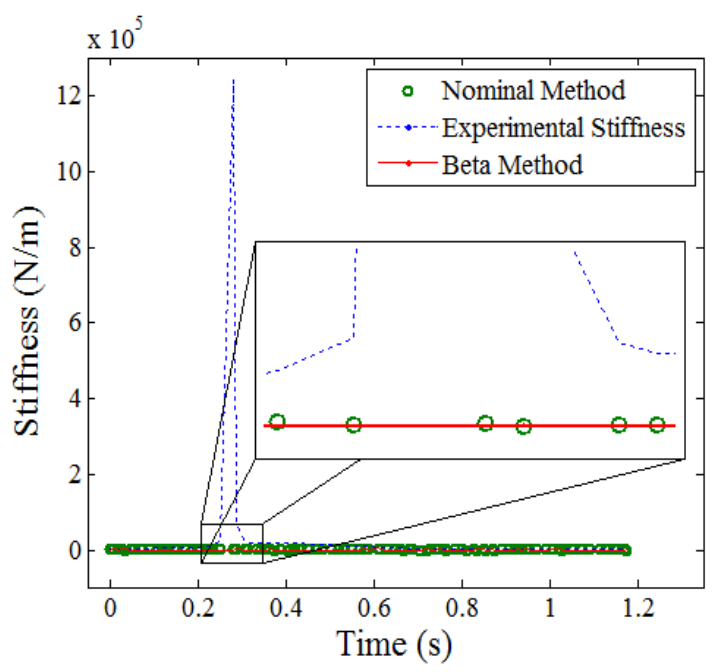

Figure 11. Example of a failed attempt at accurately estimating endpoint stiffness using two different muscle force computation methods (and HMM-predicted $\gamma$ ).

Can transient changes in endpoint stiffness be predicted? Results obtained from the prediction trials in subject 01 are displayed in Fig. 12. This subject produced a statistically significant correlation coefficient between predicted and actual stiffness values $(r=0.2561, p=0.0101<$ 
0.05). But even more impressive is the fact the predicted endpoint stiffness profile closely matches the actual experimental stiffness. Combining the nominal method along with the HMM method yields a transient peak that is more than $99 \%$ accurate compared to the actual experimental peak. This number is compared to $48.5 \%$ peak accuracy for the combined beta + HMM methods and $16.2 \%$ accuracy for the combined nominal + constant methods. Though the last combination of these approaches actually yielded the highest correlation coefficient between predicted and actual stiffness values, it is evident from current observations that correlation analysis alone does not provide the full picture of the results. These outcomes reinforce the possibility of probabilistic estimation as a valid tool for inference of stochastic processes and also suggest that modulation of the $\gamma(t)$ variable is indeed a way to directly affect changes in $\boldsymbol{\beta}(\boldsymbol{t})$, and hence endpoint stiffness, pHRI system stability, and performance.

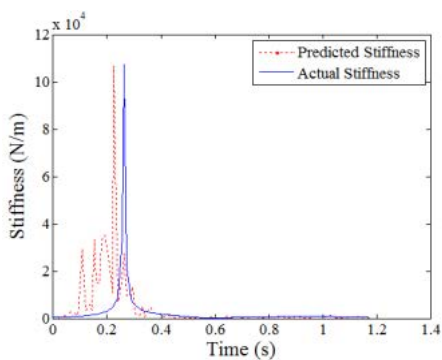

(a) Nominal + HMM methods

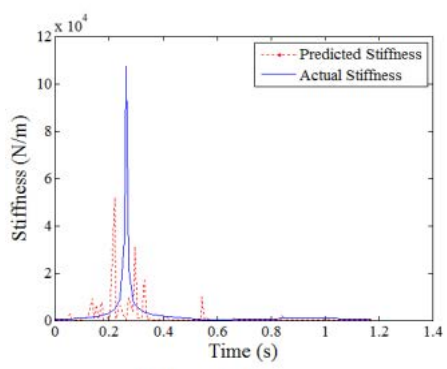

(b) Beta + HMM methods

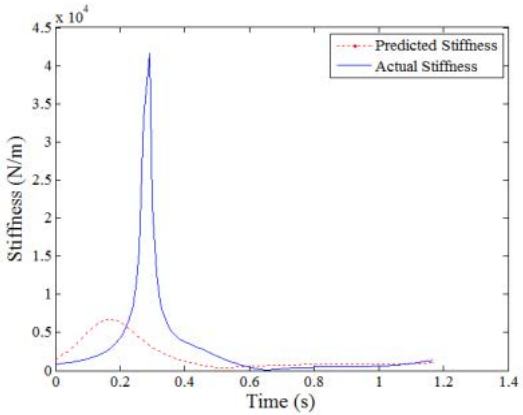

(c) Nominal + Constant methods

Figure 12. Example of prediction results from a single subject (subject 01), with successful prediction of endpoint stiffness using the nominal + HMM methods.

The time lag between predicted and actual results for subject 01 , with the predictive model ahead in the time spectrum, was also notable in some graphs of Fig. 12. The model's time lead ahead of the experimental method is an encouraging factor, suggesting that a proactive approach to stiffness compensation in haptic control systems is possible, provided real-time implementation of the current method is carried out. However, the delay between predicted and experimental stiffness plot may play a role in lowering the correlation values between the two datasets. Also, it is not well understood why this time lead also appears in the nominal + constant case. Since the value chosen for the scaling constant in this case was the mean value obtained from averaging the scaling values obtained from the nominal $+H M M$ case, it is possible that such an averaging procedure may explain the observed behavior.

Also, normalizing the predicted and actual stiffness plots with respect to their corresponding maximal values yielded mostly similar correlation results. The normalized results mostly showed a time delay between predicted and actual stiffness result, with the prediction mostly ahead (see Fig. 13 for an example). However, time shifting of the predicted data (and array padding of the missing data) did not significantly improve the correlation between the two datasets. This indicates that the 
lower stiffness values, relative to the maximal value, are not being accurately predicted either. These results suggest a stiffness scaling issue that needs to be addressed.
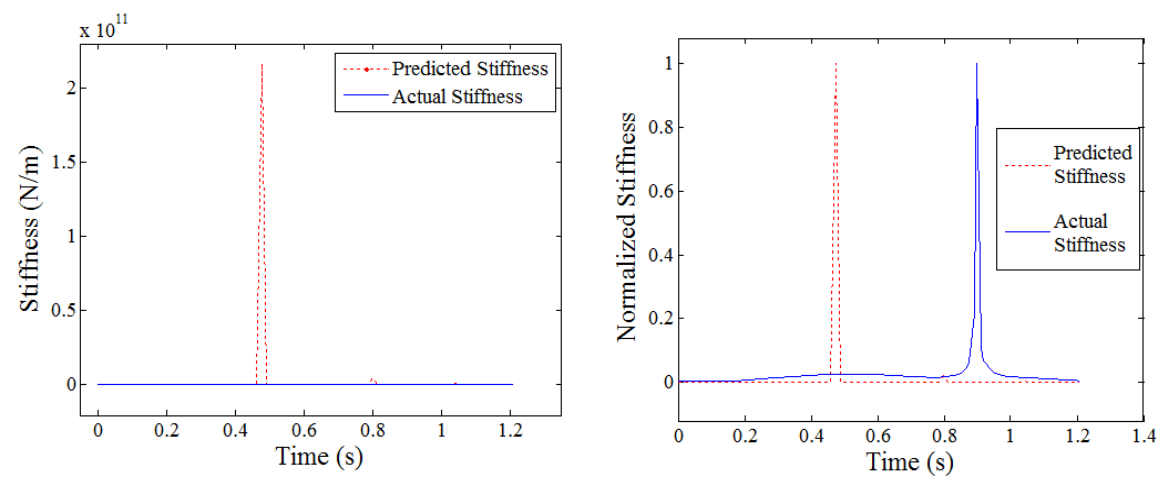

Figure 13. Example of showing stiffness prediction results before (left) and after (right) normalization of predicted and actual stiffness, with respect to their corresponding maximum values. Nominal + HMM methods were used in this case.

\section{Stiffness Input and Output Mappings}

Given the physiological boundary constraints of each muscle, the maximum endpoint stiffness produced by the Hu et al. (2011) prediction model is limited to the $10^{3}$ order of magnitude. To account for the low muscle force estimates previously mentioned, a mapping of both input (HMM training) and output (predicted result) stiffness was performed, which also resulted in the constraining the values of $\gamma(t)$ between 0 and 1. For input and output endpoint stiffness values $k_{\text {in }}$ and $k_{\text {out }}$, mapped values $\hat{k}_{\text {in }}$ and $\hat{k}_{\text {out }}$ were obtained using equations (10) and (11), respectively.

$$
\begin{gathered}
\hat{k}_{\text {in }}= \begin{cases}k_{\text {in }} & \text { if } k_{\text {in }}<1 \\
\ln \left(k_{\text {in }}\right) & \text { if } k_{\text {in }}>1\end{cases} \\
k_{\text {out }}= \begin{cases}\hat{k}_{\text {out }} & \text { if } \hat{k}_{\text {out }}<1 \\
\exp \left(\hat{k}_{\text {out }}\right) & \text { if } \hat{k}_{\text {out }}>1\end{cases}
\end{gathered}
$$

It is probable that the use of these mapping rules may have contributed to the overshoot in most stiffness estimations as well as the inability of our model to accurately predict lower stiffness values with consistency (Fig. 10).

\section{How Does the CNS Achieve Proper Modulation of Endpoint Stiffness in a Single Direction?}

Theories about how a human operator regulates endpoint impedance have been proposed for a long time. Given current knowledge of the role of antagonistic co-contraction in modulating endpoint impedance, it is necessary to hone in on what specific control processes can be used to accurately characterize how the human operator is able to affect impedance at an interaction port during pHRI. This research partly aims toward this goal, since understanding mechanisms of neuromuscular adaption is key to relating them to system stability and performance characteristics. For instance, based on the preliminary findings presented in this study, it can be suggested that Crowninshield and Brand's (1981) optimality principle, guiding the choice of cost function for muscle force computation, may only be an incomplete approach rather than anything else. Combination of this minimum energy optimality criterion approach with methods accounting for co-activation of antagonistic muscles seems to be a more plausible thought direction. The current study postulates that the nullspace of the mapping between muscle forces and joint torques, a 
multidimensional variable vector $\boldsymbol{\beta}(\boldsymbol{t})$, directly affects changes in endpoint stiffness values. However, from a control perspective, achieving prediction of the vector's numerous entries, which are more than likely co-dependent, is not a desirable task. As an alternative, the study postulates that control of a one-dimensional time- and (likely) configuration-dependent scaling factor $\gamma(t)$, assumed to be constant in previous literature, is a better approach. Table 3 displays the correlation coefficients between $\frac{1}{\gamma(t)}$ and $\|\boldsymbol{\beta}(\boldsymbol{t})\|$ for all subjects, as obtained from

Table 3. Summary of the correlation between $\frac{1}{\gamma(t)}$ and $\|\boldsymbol{\beta}(\boldsymbol{t})\|$ for all subjects, as obtained from analyzing the training data in the beta + $H M M$ case, with statistically significant results highlighted in bold.

\begin{tabular}{|c|c|c|c|c|c|c|c|c|c|c|}
\hline $\begin{array}{c}\text { Subject } \\
\text { Number }\end{array}$ & 00 & 01 & 02 & 03 & 04 & 05 & 06 & 07 & 08 & 09 \\
\hline Correlation & 0.1958 & $\mathbf{0 . 5 1 8 8}$ & $\mathbf{0 . 4 0 9 6}$ & $\mathbf{0 . 9 0 1 5}$ & 0.0996 & 0.0114 & -0.0793 & $\mathbf{0 . 4 4 2 2}$ & $\mathbf{0 . 4 4 2 9}$ & $\mathbf{0 . 2 7 7 4}$ \\
\hline p value & 0.0509 & $\mathbf{4 . 4 5 E - 0 8}$ & $\mathbf{0 . 0 0 1}$ & $\mathbf{3 . 6 6 E - 0 4}$ & 0.5005 & 0.9169 & 0.4628 & $\mathbf{2 . 2 7 E - 0 5}$ & $\mathbf{6 . 2 3 E - 0 6}$ & $\mathbf{0 . 0 0 7 4}$ \\
\hline
\end{tabular}

analyzing the training data in the beta $+H M M$ case. At least 6 out of 10 subjects exhibited statistically significant correlations between the 2 variables. Eq. (4) characterizes the nature of the relationship between the two variables, suggesting a possible mapping between the inverse of the dimensional scaling factor $\frac{1}{\gamma(t)}$ and the vector $\boldsymbol{\beta}(\boldsymbol{t})$. Examination of the relationship between these two variables is shown by the normalized time realizations in Fig. 14, as well as the normalized histogram distributions in Fig. 15, which show similar shape and skewness. Based on these observations, the highly discrepant and non-statistically significant results obtained in the beta + $H M M$ case can be attributed to the fact that this combination is redundant. Such results could have been expected, given the hypothesized interdependence of the two variables, $\frac{1}{\gamma(t)}$ and $\|\boldsymbol{\beta}(\boldsymbol{t})\|$.

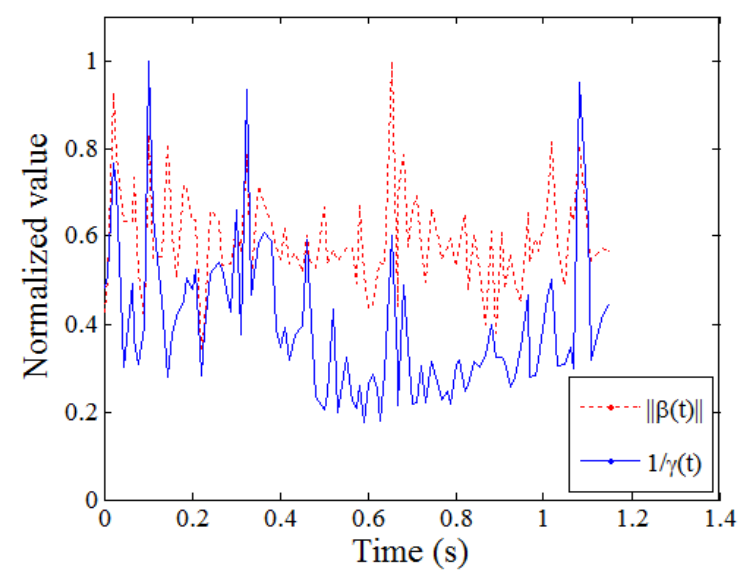

Figure 14. Comparison of normalized time realizations of $\|\beta(t)\|$ and $\frac{1}{\gamma(t)}$ in a single subject (subject 01) shows a similar shape.

Therefore, using the standard optimality principle to generate muscle forces given a certain arm configuration and endpoint force, we suggest that the CNS is able to tune the endpoint impedance characteristics in a single direction by additionally modulating a single scaling parameter, rather than a multivariable factor. 


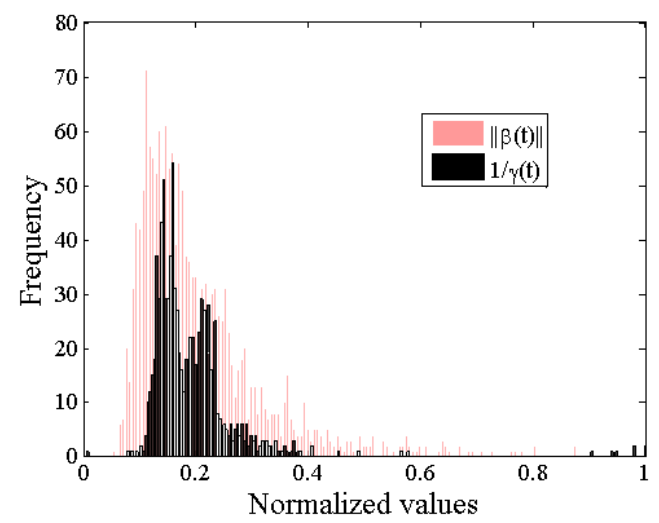

Figure 15. Comparison of histogram distributions of $\|\beta(t)\|$ and $\frac{1}{\gamma(t)}$ show a similar shape and skewness.

\section{Limitations and Future Work}

Results presented in this study currently only apply to 1-DOF systems. Directional tuning of endpoint impedance was not taken into account, due to the assumption that both force and endpoint displacement vectors in the experiments vary along the same line of action. This assumption is made by considering that endpoint displacements along the radial direction of the haptic paddle device in Fig. 4 are negligible. However, the endpoint stiffness estimation method outlined in Fig. 2 produces a $6 \times 6$ endpoint stiffness matrix that can be used to analyze both value-based and directional effects of changes in model parameters on the size and shape of the stiffness ellipsoid, but there would be no experimental counterpart to the simulated data, since our current device is a 1-DOF system. The current study only examined the second diagonal component of the stiffness matrix by assuming that the effects of other components were negligible, due to 1-DOF displacement only.

The paper also assumes that inertia and damping contributions to endpoint impedance are negligible in this case, since stiffness is thought to be especially important during posture maintenance (Hu et al., 2011). In some previous literature, estimate of joint stiffness, independent of inertia and damping, was obtained by considering only the portion of the perturbation response around peak displacement, where acceleration and velocity are low enough (Franklin \& Milner, 2003). However, Konczak, Brommann, and Kalveram (1999) showed that greater accuracy was achieved when predicting position trajectories of subjects performing hold tasks using a full model, including both variable stiffness and damping parameters, as compared to reduced models neglecting damping. Changes in stiffness and damping were also found to be significant during the braking phase of the movement. Based on this consideration, exploration of damping effects on our system would be necessary. Future work in this research will also include the generalization of results to higher degrees of freedom by building a multidimensional test bed device for proof of concept of a controller that tunes its impedances gains according to stochastic changes in operator physical state.

In addition, the perturbation force amplitude range $(0-40 \mathrm{~N})$ in this paper is higher than the ones found in previous literature (e.g., 6.5-9.5N in Selen, Franklin, \& Wolpert, 2009). It is likely that our nominal + constant type trials, which essentially use the same approach found in Hu et al. (2011), suffer from the same limitation of low stiffness estimation. Hence, the model may be better suited for low-range stiffness prediction. Additionally, testing of the model with maximal muscle activation values at different configurations yielded endpoint stiffness values that were found to be lower than the range of stiffness values calculated from the experimental set-up. 
However, because some successful trials were found using the nominal $+H M M$ approach, it remains to be investigated whether the inconsistency in the results stems from a higher range of force perturbation or from non-optimal HMM training parameters.

Sampling variance during data collection during experimental design may have also played a great role in the performance of the HMM method in particular, given the effect of both state and observation sequence lengths on the accuracy of the predictive model. Also, convergence during training of the predictor models was not always immediately achieved and required multiple trials among the randomly selected initial state distributions to reach a local minimum in the optimization during HMM training. Choice of optimal model parameters, in order to improve and find a balance between prediction accuracy, train time, and test time, will also be conducted in the near future to ensure repeatability of the promising results outlined in this study.

Implementation of the currently proposed method into the design of a novel haptic controller will require drastic reduction in computation time for both online estimation and proactive gain scheduling. Currently, the most computationally expensive steps in the prediction process stem from calculation of joint data and inertial parameters for a given configuration. Translation of the current code to run on target devices as a binary file will drastically reduce the computational burden and allow us to evaluate prediction method performance in real-time.

\section{Conclusion}

In the current study, we proposed a new approach to quasi-static endpoint stiffness estimation in 1-DOF haptic control systems, using a combination of probabilistic estimation of hidden variables believed to directly affect endpoint stiffness with a stiffness prediction method previously outlined in $\mathrm{Hu}$ et al. (2011). The proposed approach was intended to achieve true estimation of absolute stiffness level values by accounting for muscle co-contraction, in order to improve upon lower estimation issues encountered in previous literature. Incorporation of operator dynamics into the pHRI system was performed using a musculoskeletal model of the upper right limb, developed by Ueda et al. (2010).

The study hypothesized that endpoint stiffness estimation could be achieved through probabilistic estimation of a multidimensional parameter $\boldsymbol{\beta}(\boldsymbol{t})$ known to represent the mapping kernel between muscle forces and joint torques (i.e., muscle co-activation). The estimation was ultimately performed not through direct modulation of the parameter itself, but through probabilistic prediction of a one-dimensional scaling factor $\gamma(t)$ shown to be related to the multidimensional variable, by means of hidden Markov model (HMM) prediction theory. Results show statistically significant correlation between predicted and actual stiffness values in a third of the total trials. Impressive prediction results were found in a single subject, using a combination of the proposed method along with the standard static optimization method for muscle force computation (Crowninshield \& Brand, 1981), compared to other combinations. The possibility of successful prediction using one of the proposed methods was shown, although consistent performance requires evaluation of the best HMM parameters needed to run the simulations.

Insights obtained from the current study about how the CNS is able to achieve modulation of operator endpoint impedance indicate that real-time optimization of a single one-dimensional and time-varying parameter, in addition to the static optimization of the muscle force vector, can allow for incorporation of muscle co-contraction effects on changes in endpoint stiffness along a single direction.

Future work in this study will ensure repeatability of the current findings, evaluation of approach robustness to slight input changes, and the generalization of the proposed approach to multi-DOF systems. The ultimate goal of this research is to design a novel haptic controller that tunes its impedance gains according to stochastic changes in operator physical state (endpoint impedance) and intent. Benefits of this work include contributions to communities interested in novel adaptive shared control approaches for advanced manufacturing and process design (e.g., automobile, aerospace, and construction industries). 


\section{Acknowledgements}

The authors would like to acknowledge the National Science Foundation for their sponsorship (National Robotics Initiative Grant Number 1317718 and Computing Research Infrastructure Grant Number 1059362) and all members of the Bio-Robotics \& Human Modeling Laboratory at the Georgia Institute of Technology. Opinions expressed in this document can be attributed to the authors alone and do not necessarily reflect the views of either institution.

\section{References}

Bernstein, N. A. (1967, originally published in 1940). The coordination and regulation of movements. Oxford, UK: Pergamon Press.

Burdet, E., Osu, R., Franklin, D., Yoshioka, T., Milner, T., \& Kawato, M. (2000). A method for measuring endpoint stiffness during multi-joint arm movements. Journal of Biomechanics 33(12), 1705-1709.

Collins, J. (1995). The redundant nature of locomotor optimization laws. Journal of Biomechanics 28(3), 251-267.

Crowninshield, R. D., \& Brand, R. A. (1981), A physiologically based criterion of muscle force prediction in locomotion. Journal of Biomechanics 14(11), 793-801.

Cui, L., Perreault, E. J., Maas, H., \& Sandercock, T. G. (2008). Modeling short-range stiffness of feline lower hindlimb muscles. Journal of Biomechanics 41(9), 1945-1952.

Darainy, M., Malfait, N., Gribble, P. L., Towhidkhah, F., \& Ostry, D. J. (2004). Learning to control arm stiffness under static conditions. Journal of Neurophysiology 92(6), 33443350 .

Flash, T., \& Mussa-Ivaldi, F. (1990). Human arm stiffness characteristics during the maintenance of posture. Experimental Brain Research 82(2), 315-326.

Franklin, D. W., Liaw, G., Milner, T. E., Osu, R., Burdet, E., \& Kawato, M. (2007). Endpoint stiffness of the arm is directionally tuned to instability in the environment. The Journal of Neuroscience 27(29), 7705-7716.

Franklin, D. W., \& Milner, T. E. (2003). Adaptive control of stiffness to stabilize hand position with large loads. Experimental Brain Research, 152(2), 211-220.

Gallagher, W., McPherson, T., Huggins, J., Shinohara, M., Gao, D., Menassa, R., \& Ueda, J. (2012). An improved human-robot interface by measurement of muscle stiffness. In Proceedings of the 4th IEEE RAS \& EMBS International Conference on Biomedical Robotics and Biomechatronics (BioRob) (pp. 177---182). doi:10.1109/BioRob.2012.6290814

Gallagher, W. J. (2013). Modeling of operator action for intelligent control of haptic human-robot interfaces (Doctoral Thesis). Atlanta, GA: Georgia Institute of Technology. Retrieved from http://hdl.handle.net/1853/50258

Garner, B. A., \& Pandy, M. G. (2003). Estimation of musculotendon properties in the human upper limb. Annals of Biomedical Engineering 31(2), 207-220.

Gomi, H., \& Osu, R. (1998). Task-dependent viscoelasticity of human multijoint arm and its spatial characteristics for interaction with environments. The Journal of Neuroscience 18(21), 8965-8978.

Gribble, P. L., Mullin, L. I., Cothros, N., \& Mattar, A. (2003). Role of cocontraction in arm movement accuracy. Journal of Neurophysiology 89(5), 2396-2405.

Hogan, N. (1984). Adaptive control of mechanical impedance by coactivation of antagonist muscles. IEEE Transactions on Automatic Control 29(8), 681-690. 
Holzbaur, K. R., Murray, W. M., \& Delp, S. L. (2005). A model of the upper extremity for simulating musculoskeletal surgery and analyzing neuromuscular control. Annals of Biomedical Engineering 33(6), 829-840.

Hu, X., Murray, W. M., \& Perreault, E. J. (2011). Muscle short-range stiffness can be used to estimate the endpoint stiffness of the human arm. Journal of Neurophysiology 105(4), $1633-1641$.

Konczak, J., Brommann, K., \& Kalveram, K. T. (1999). Identification of time-varying stiffness, damping, and equilibrium position in human forearm movements. Motor Control, 3(4), 394-413.

Krutky, M. A., Ravichandran, V. J., Trumbower, R. D., \& Perreault, E. J. (2010). Interactions between limb and environmental mechanics influence stretch reflex sensitivity in the human arm. Journal of Neurophysiology 103(1), 429-440.

Krutky, M. A., Trumbower, R. D., \& Perreault, E. J. (2013), Influence of environmental stability on the regulation of end-point impedance during the maintenance of arm posture. Journal of Neurophysiology 109(4), 1045-1054.

Maganaris, C. N., \& Paul, J. P. (2002). Tensile properties of the in vivo human gastrocnemius tendon. Journal of Biomechanics 35(12), 1639-1646.

Magnusson, S. P., Aagaard, P., Rosager, S., Dyhre-Poulsen, P., \& Kjaer, M. (2001). Loaddisplacement properties of the human triceps surae aponeurosis in vivo. The Journal of Physiology 531(1), 277-288.

McIntyre, J., Mussa-Ivaldi, F., \& Bizzi, E. (1996). The control of stable postures in the multijoint arm. Experimental Brain Research 110(2), 248-264.

Milner, T. E., Cloutier, C., Leger, A. B., \& Franklin, D. W. (1995). Inability to activate muscles maximally during cocontraction and the effect on joint stiffness. Experimental Brain Research 107(2), 293-305.

Moualeu, A., Gallagher, W., \& Ueda, J. (2014). Support vector machine classification of muscle cocontraction to improve physical human-robot interaction. In Proceedings of the IEEE/RSJ International Conference on Intelligent Robots and Systems (IROS). doi:10.1109/IROS.2014.6942852

Mussa-Ivaldi, F. A., Hogan, N., \& Bizzi, E. (1985). Neural, mechanical, and geometric factors subserving arm posture in humans. The Journal of Neuroscience 5(10), 2732-2743.

Oliver, N., \& Horvitz, E. (2005). A comparison of HMMs and dynamic Bayesian networks for recognizing office activities. In Proceedings of the $10^{\text {th }}$ International Conference on User Modeling (pp. 199-209). Edinburgh, Scotland, UK: Springer.

Osu, R., Franklin, D. W., Kato, H., Gomi, H., Domen, K., Yoshioka, T., \& Kawato, M. (2002). Short-and long-term changes in joint co-contraction associated with motor learning as revealed from surface EMG. Journal of Neurophysiology, 88(2), 991-1004.

Patel, H., O'Neill, G., \& Artemiadis, P. (2014). On the effect of muscular cocontraction on the 3D human arm impedance. IEEE Transactions on Biomedical Engineering 61(10), 2602-2608.

Perreault, E. J., Kirsch, R. F., \& Crago, P. E. (2002). Voluntary control of static endpoint stiffness during force regulation tasks. Journal of Neurophysiology 87(6), 2808-2816.

Perreault, E. J., Kirsch, R. F., \& Crago, P. E. (2001). Effects of voluntary force generation on the elastic components of endpoint stiffness. Experimental Brain Research 141(3), 312-323.

Rabiner, L. (1989). A tutorial on hidden Markov models and selected applications in speech recognition. Proceedings of the IEEE 77(2), 257-286.

Rabiner, L., \& Juang, B.-H. (1986). An introduction to hidden Markov models. ASSP Magazine 3(1), 4-16. New York, NY: IEEE. 
Schmid, A. J., Weede, O., \& Worn, H. (2007). Proactive robot task selection given a human intention estimate. In Proceedings of the 16th IEEE Conference on Robot and Human Interactive Communication (RO-MAN) (pp. 726-731). doi:10.1109/ROMAN.2007.4415181

Selen, L. P., Franklin, D. W., \& Wolpert, D. M. (2009). Impedance control reduces instability that arises from motor noise. The Journal of Neuroscience, 29(40), 12606-12616.

Takeda, T., Hirata, Y., \& Kosuge, K. (2007). Dance step estimation method based on HMM for dance partner robot. IEEE Transactions on Industrial Electronics 54(2), 699-706.

Thoroughman, K. A., \& Shadmehr, R. (1999). Electromyographic correlates of learning an internal model of reaching movements. The Journal of Neuroscience 19(19), 8573-8588.

Tsuji, T., \& Kaneko, M. (1996). Estimation and modeling of human hand impedance during isometric muscle contraction. In Proceedings of the ASME Dynamic Systems and Control Division (DSC) (pp. 575-582). Atlanta, GA.

Ueda, J., Ming, D., Krishnamoorthy, V., Shinohara, M., \& Ogasawara, T. (2010). Individual muscle control using an exoskeleton robot for muscle function testing. IEEE Transactions on Neural Systems and Rehabilitation Engineering 18(4), 339-350.

Yamada, Y., Umetani, Y., Daitoh, H., \& Sakai, T. (1999). Construction of a human/robot coexistence system based on a model of human will-intention and desire. In Proceedings of the IEEE International Conference on Robotics and Automation (pp. 2861-2867). doi:10.1109/ROBOT.1999.774031

Zajac, F. E. (1988). Muscle and tendon: Properties, models, scaling, and application to biomechanics and motor control. Critical Reviews in Biomedical Engineering 17(4), 359-411.

Antonio Moualeu, Bio-Robotics \& Human Modeling Laboratory at the Georgia Institute of Technology, Atlanta, GA USA. Email: amoualeu3@mail.gatech.edu; Jun Ueda, Bio-Robotics \& Human Modeling Laboratory at the Georgia Institute of Technology, Atlanta, GA USA. Email: jun.ueda@me.gatech.edu 